\title{
Associations of Objective Physical Activity and Sedentary Behavior with Frailty in Community-dwelling Older Adults: A Mini-review
}

\author{
Si Chen ${ }^{1}$, Tao Chen ${ }^{2}$, Shuzo Kumagai ${ }^{1,3 *}$, Hyuntae Park $^{2 *}$ \\ ${ }^{1}$ Graduate School of Human-Environment Studies, Kyushu University, Fukuoka, Japan; ${ }^{2}$ Department of Health Care Science, Dong-A University, Busan, Korea; ${ }^{3}$ Center for \\ Health Science and Counseling, Kyushu University, Fukuoka, Japan
}

PURPOSE: This review aimed to evaluate the relationship between objectively measured physical activity and sedentary behavior and frailty in community-dwelling older adults.

METHODS: An electronic search was undertaken using the MEDLINE ${ }^{\circledR}$ databases from January 1 st 2010 to December 31 st 2019. Titles, abstracts, and full texts of the studies identified by the search were scrutinized by the author to determine eligibility for an inclusion in this review.

RESULTS: The search identified 87 relevant articles resulted, of which eight studies from four cohorts met the inclusion requirements. Almost all the studies reported a significantly negative association between moderate-to-vigorous physical activity (MVPA) and frailty, while only one was a longitudinal study. Significant associations between sedentary time (ST) and higher severity of frailty were observed, however, the findings of associations of sedentary bouts were mixed.

IMPLICATION: Higher amounts of ST and lower amounts of MVPA were associated with a higher prevalence of frailty or worse frailty levels. Replacing ST with MVPA may offset the detrimental consequences of ST on frailty. More longitudinal studies and quantitative researches are needed to investigate the association of MVPA and ST bouts with frailty.

Key words: Frailty; Physical activity; Sedentary behavior; Objective measures; Community-dwelling older adults

\section{INTRODUCTION}

Population aging is accelerating worldwide, with 962 million people older than 65 years in 2017, and this number is estimated to reach 2 billion people by 2050, which has profound implications for the planning and delivery of health and social care [1]. Frailty, one of the most problematic expressions of aging, is defined as a medical syndrome with multiple causes and contributors characterized by diminished strength, endurance, and reduced physiologic function and increases an individual's risk of dependency and/or death [2]. To date, numerous instruments were designed to screen and assess frailty status, while a marked hetero- geneity was found between these frailty instruments [3]. The varied definitions for frailty may cause substantial variation in the reported prevalence of frailty from $4.0 \%$ to $59.1 \%$ [4]. Nevertheless, the associations between frailty and increased risk of adverse outcomes such as all-cause mortality [5], hospitalization [6], future falls [7], disability [8], cognitive function [9], and onset of cardiovascular disease [10] have been clearly demonstrated by recent systematic reviews. Indeed, it is notable that frailty is a dynamic condition and frailty status can shift between better and worse over time [11]. This aspect of frailty may present an opening for potential preventative and restorative interventions.

Lifestyle is considered one of the main keystones in the development 
of frailty, and a healthy lifestyle can help older adults to manage frailty [12]. As a common component of lifestyle, daily physical activity (PA) and sedentary behavior (SB) are closely related to health outcomes. Replacing SB with PA may have some beneficial effects on adverse outcomes such as all-cause mortality [13], sarcopenia [14], and poor physical function [15] in older adults. Therefore, daily PA and SB may also play an important role in the development of frailty since frailty is the result of the dysregulation of the well-tuned complex dynamical system of a resilient organism while PA can simultaneously up-regulate many systems to a higher functional level [16]. Recent reviews summarized the evidence regarding the association of daily PA and SB with frailty [17, 18], however, most studies used subjective methods, which varied among studies, to assess PA and SB. It is notable that subjective measures are usually reported to either over- or underestimate PA levels due to recall bias, particularly in older adults [19]. The heterogeneity of the assessment methods of PA and SB and different population settings in these two systematic reviews may limit the generalizability of their findings. The use of accelerometer allows objective and more accurate assessment of PA and SB, as well as detailed patterns of accumulation which may play an important role in the progress of frailty. However, to our best knowledge, a review has not been conducted to identify the associations between objectively measured PA and SB and frailty in community-dwelling older adults. Summarizing the existing evidence using objectively measured data will enable researchers and healthcare providers to better understand the associations between PA and SB and frailty. Therefore, in the present study, we review the epidemiological studies which exam- ined the relationship between objectively measured PA and SB with frailty in community-dwelling older adults.

\section{METHODS}

An electronic search was undertaken using the MEDLINE ${ }^{\circledR}$ databases from 1st January 2010 to 31st December 2019. The search employed the terms 'frailty' or 'frail' combined with the terms 'physical activity' or 'sedentary behavior', details were as follows: Physical activity [Title] or Light Physical Activity [Title] or Moderate-Vigorous Physical Activity [Title] or Bouts $<10$ minutes [Title] or Bouts $\geq 10$ minutes [Title] or Sedentary behavior [Title] or Sedentary time [Title] or Sedentary bout [Title] or Sedentary break [Title], and Frailty [Title] or Frail [Title]. Titles, abstracts, and full texts of the studies identified by the search were scrutinized by the author Chen $\mathrm{Si}$ to determine eligibility for inclusion in the present review. Any studies providing or potentially capable of providing cross-sectional or longitudinal data regarding the association between objectively measured PA and SB and frailty in community-dwelling older adults were eligible. Studies including non-English, randomized controlled trial, review, comment, selected samples (subjects with diseases), and subjectively measured PA or SB were excluded. Moreover, when multiple studies used the same cohort, only the study with the largest sample size was included. The following data were extracted from each study: study design, author, cohort name, sample size, age, PA, and SB assessment method, frailty instrument, statistical analysis, adjustment variable, and effect estimate.

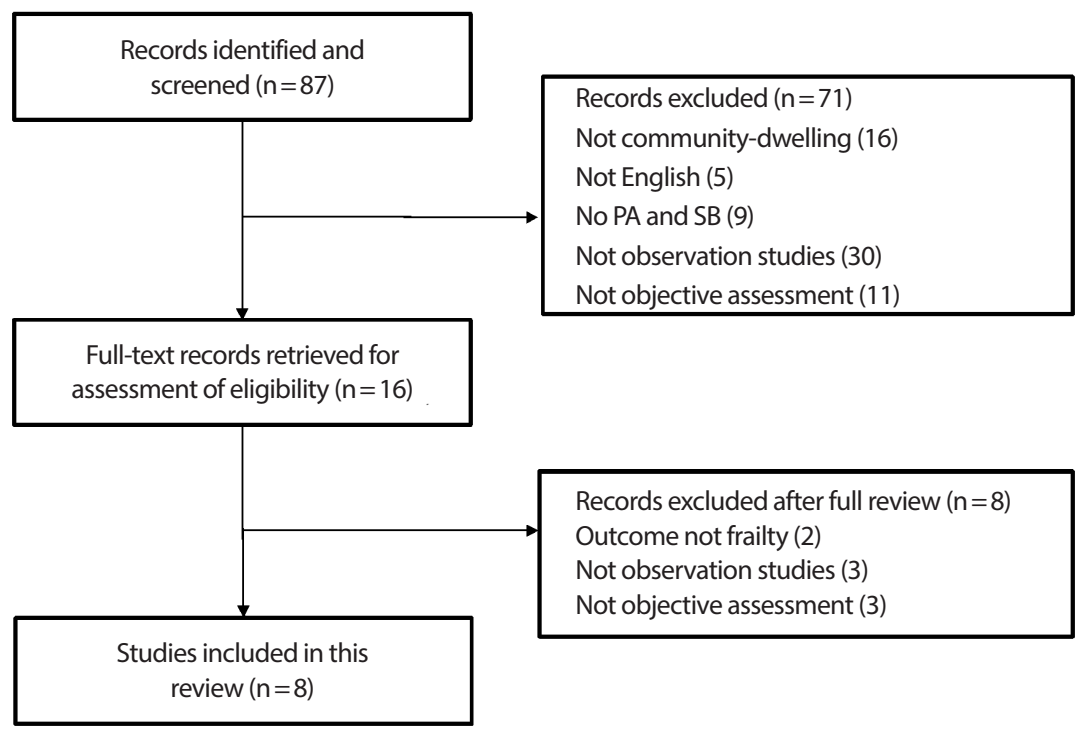

Fig. 1. Flowchart of studies included in review. 


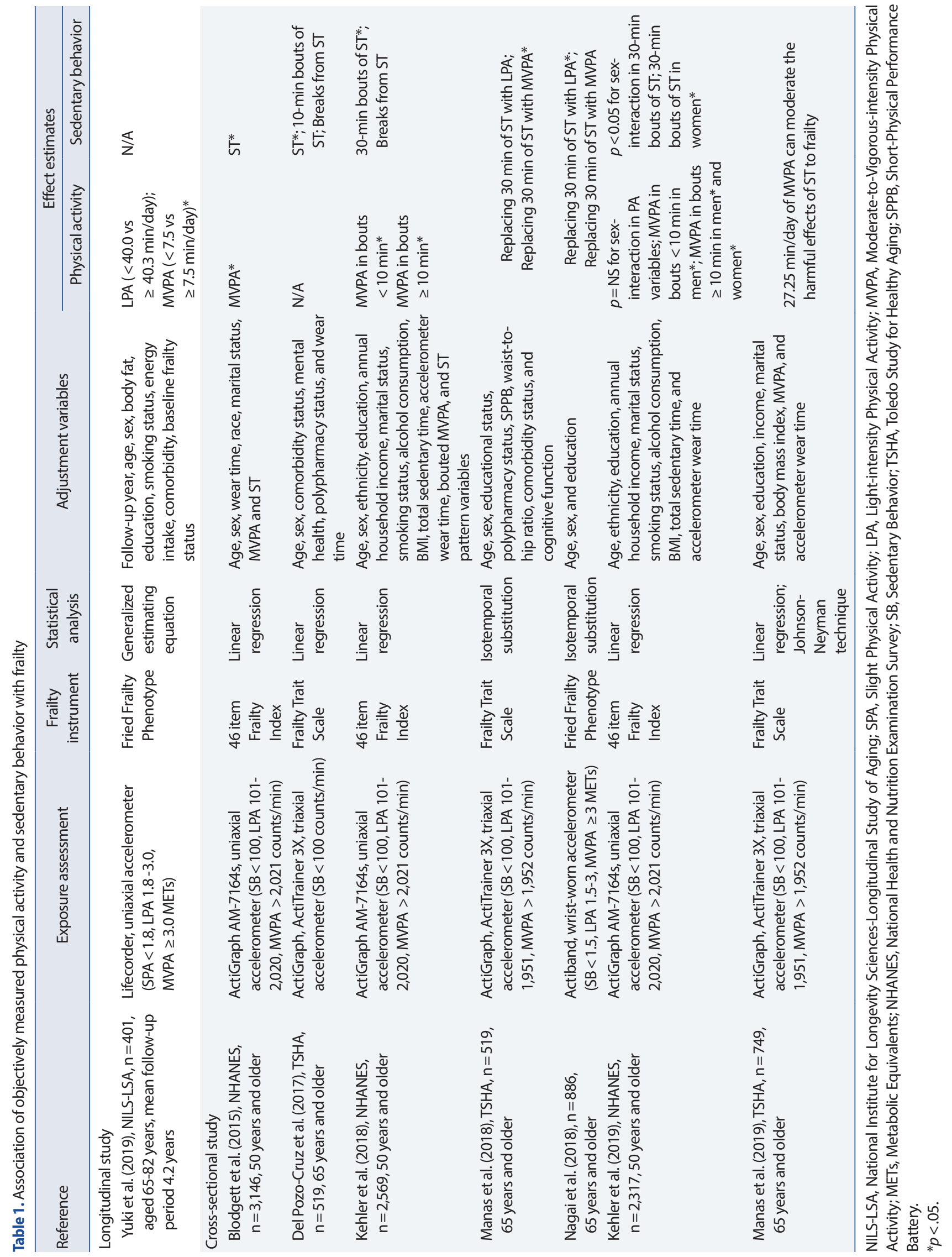




\section{RESULTS}

The search identified 87 relevant articles (Fig. 1). Of these 87 references, 71 were excluded through title and abstract screening. Most of the articles were excluded at this stage because: 1) The study design was not observational ( $\mathrm{n}=30)$; 2 ) The study population was not communitydwelling older adults $(\mathrm{n}=16)$, or 3$)$ Studies did not report PA and SB or using a subjective way to assess PA and SB $(n=20)$. Sixteen studies were left for full-text review, of which eight studies were excluded because: 1) The outcome was not frailty $(\mathrm{n}=2), 2)$ The study design was not observational $(\mathrm{n}=3)$, or 3) Using a subjective method to assess PA and SB $(n=3)$, resulting eight from four cohorts meeting the inclusion requirements for the present review [20-27].

The characteristics of the eight included studies are summarized in Table 1. Of these studies, only one was a longitudinal study with a mean follow-up period of 4.2 years [27] and others were cross-sectional studies [20-26]. Three studies were from the United States [20,22,23], 3 from Spain $[21,24,25]$, and 2 from Japan [26,27]. Although some studies used data from the same cohorts, such as from the Toledo Study of Health Aging (TSHA, 3 times) [21,24,25] and National Health and Nutrition Examination Study (NHANES, 3 times) [20,22,23], the exposure variables were analyzed differently. The sample sizes of these studies ranged from 401 to 3,146, with mean age from 63.3 to 78.8 years. All studies recruited men and women, with approximately 44.4 to $70.0 \%$ were women, while only one study reported the sex-specific results [23]. PA and SB were all measured in an objective way, but different devices were used in each cohort. National Institute for Longevity Sciences-Longitudinal Study of Aging (NILS-LSA) and NHANES used uniaxial accelerometers [20,22,23,27], TSHA used triaxial accelerometers [21,24,25], and Nagai et al. used wrist-worn accelerometers [26]. Two definitions of PA and SB in these studies were observed: NHANES and TSHA used 'counts/min' and similar cut-off values to define SB, LPA, and MVPA [20-25], while NILS-LSA and Nagai et al. used "METs" but different cut-off values to define SB and LPA $[26,27]$. Three different frailty instruments were used to screen frailty status including Fried Frailty Phenotype [26,27], 46-item Frailty Index [20,22,23], and Frailty Trait Scale [21,24,25]. As for the statistical analysis, the most common method was linear regression analysis, however, the variables adjusted in the models varied widely among studies even in the same cohort. Two studies used a new approach, the isotemporal substitution model, to investigate the effect of replacing 30 min of SB with LPA and MVPA on frailty status $[24,26]$. Moreover, one study used the Johnson-Neyman technique to elucidate the cut-off value of MVPA by which moderates the harmful effects of ST to frailty [25].

The NILS-LSA [27] reported the longitudinal association between MVPA and Fried Frailty Phenotype. By modeling the MVPA time as a categorical exposure ( $<7.5 \mathrm{vs} \geq 7.5 \mathrm{~min} /$ day) and frailty as a categorical outcome, participants who accumulated the lower amount of MVPA had odds ratios (OR) (95\% confidence interval [CI]) of 1.80 (1.05-3.09; $P$ $<.05)$ for a development of frailty at follow-up. However, this result did not observe significant association between LPA (<40.0 vs $\geq 40.3 \mathrm{~min} /$ day) and frailty. When using an isotemporal substitution analysis, both the TSHA [23] and Nagai et al. [26] found LPA and MVPA were negatively associated with frailty in the partition factor model. The NHANES [20] found MVPA time was independently associated with the 46-item Frailty Index score, and 1 hour/day of MVPA increment was related to a decreased frailty index score of 0.045. Moreover, in another two studies which also using the NHANES data [22,23], the results showed that irrespective of whether MVPA in bouts $<10$ or $\geq 10$ minutes, higher levels of MVPA were associated with reduced risk of frailty, although the association may be weakened in women. On the other hand, both the NHANES [20] and TSHA [21] found significant associations between sedentary time (ST) and higher severity of frailty. Moreover, the study using data from the NHANES [22] found 30-minute bouts of ST was significantly related to an incensement of the frailty index score, while such a relationship was not observed between 10-minute bouts of ST and Frailty Trait Scale score in the TSHA [21]. In addition, 30-minute bout of ST was associated with worse frailty in women but not men [23] When comprehensively examined the association of PA and SB with frailty, replacing ST with higher-intensity activity could lower the severity of frailty, however, the results were inconsistent between studies. TSHA [24] found substituting ST with MVPA but not LPA was associated with positive effects on frailty, while Nagai et al. [26] observed an opposed result. Moreover, in the TSHA [25], the authors using the Johnson-Neyman technique and found $27.25 \mathrm{~min} /$ day of MVPA may offset the detrimental consequences of ST on frailty.

\section{IMPLICATION}

This is the first review to determine the associations of objectively measured PA and SB with frailty in community-dwelling older adults. The majority of studies included in this review observed higher amounts of ST and lower amounts of MVPA measured using accelerometer were 
associated with a higher prevalence of frailty or worse frailty levels, which confirm the findings from two previous reviews [17,18]. In addition, added new findings to the current literature. For example, prolonged ST (30 minutes) was more detrimentally associated with frailty in women than men [23] and the detrimental consequences of ST might be offset by MVPA at a cut-off of $27.25 \mathrm{~min} /$ day [25]. These findings in this review provide unique insights into the association of $\mathrm{PA}$ and $\mathrm{SB}$ with frailty which may inform future feasible approaches to managing frailty in community-dwelling older adults. However, there are also some limitations that may limit the generalizability of our findings. First, although the inclusion criterion in the present review was restricted to objectively measured PA and SB and community-dwelling aging population in order to decrease the heterogeneity between studies, some differences still exist such as the PA and SB assessment methods (triaxial vs uniaxial; wrist-worn vs waist-worn; PA and SB definition), frailty instruments (Fried Frailty Phenotype vs Frailty Index vs Frailty Trait Scale), and inconsistency of adjustment variables. These heterogeneities make some challenges for comparison of the independent associations across studies. Second, almost all these findings were obtained from cross-sectional studies (7 of 8), which precludes the ability to examine the predictive ability to make causal inferences. Third, some findings are only based on a single study such as the effects of MVPA bouts (bouts in $<10$ or $\geq$ 10 minutes) and the sex-specific associations of PA and SB with frailty. Thus, in the future, longitudinal studies using similar PA and SB assessment tools and frailty instrument are needed to investigate how patterns of PA and SB predict frailty and whether PA is associated with frailty independent of SB, and vice versa to confirm these findings. Moreover, longitudinal studies are also needed to examine whether high levels of PA attenuate or eliminate the detrimental association of SB with frailty. Lastly, the sex-differences of association between objective PA and SB and frailty should be further clarified.

\section{CONFLICTS OF INTEREST}

The authors have no conflicts of interest to declare.

\section{AUTHOR CONTRIBUTION}

Conceptualization: SC, TC, HP; Formal analysis: SC; Methodology: SC; Project administration: HP, SK; Visualization: HP; Writing - original draft: SC, TC, SK; Writing - review \& editing: TC, SK, HP.

\section{ORCID}
Si Chen
https://orcid.org/0000-0002-6041-1528
Tao Chen
https://orcid.org/0000-0002-1153-3406
Shuzo Kumagai https://orcid.org/0000-0002-4098-3385
Hyuntae Park
https://orcid.org/0000-0002-1976-0005

\section{REFERENCES}

1. World Population Prospects: the 2017 revision. New York: United Nations, Department of Economic and Social Affairs, Population Division; 2017. Available from: https://population.un.org/wpp.

2. Morley JE, Vellas B, van Kan GA, Anker SD, Bauer JM, et al. Frailty consensus: a call to action. J Am Med Dir Assoc. 2013;14(6):392-7.

3. Aguayo GA, Donneau AF, Vaillant MT, Schritz A, Franco OH, et al Agreement between 35 published frailty scores in the general population. Am J Epidemiol. 2017;186(4):420-34.

4. Collard RM, Boter H, Schoevers RA, Oude Voshaar RC. Prevalence of frailty in community-dwelling older persons: a systematic review. J Am Geriatr Soc. 2012;60(8):1487-92

5. Chang SF, Lin PL. Frail phenotype and mortality prediction: a systematic review and meta-analysis of prospective cohort studies. Int J Nurs Stud. 2015;52(8):1362-74.

6. Kojima G. Frailty as a predictor of hospitalisation among communitydwelling older people: a systematic review and meta-analysis. J Epidemiol Community Health. 2016;70(7):722-9.

7. Kojima G. Frailty as a predictor of future falls among communitydwelling older people: a systematic review and meta-analysis. J Am Med Dir Assoc. 2015;16(12):1027-33.

8. Vermeiren S, Vella-Azzopardi R, Beckwee D, Habbig AK, Scafoglieri A, et al. Frailty and the prediction of negative health outcomes: a metaanalysis. J Am Med Dir Assoc. 2016;17(12):1163 e1-e17.

9. Kojima G, Taniguchi Y, Iliffe S, Walters K. Frailty as a predictor of Alzheimer disease, vascular dementia, and all dementia among community-dwelling older people: a systematic review and meta-analysis. J Am Med Dir Assoc. 2016;17(10):881-8.

10. Veronese N, Cereda E, Stubbs B, Solmi M, Luchini C, et al. Risk of cardiovascular disease morbidity and mortality in frail and pre-frail older adults: Results from a meta-analysis and exploratory meta-regression analysis. Ageing Res Rev. 2017;35:63-73.

11. Lee JS, Auyeung TW, Leung J, Kwok T, Woo J. Transitions in frailty 
states among community-living older adults and their associated factors. J Am Med Dir Assoc. 2014;15(4):281-6.

12. Feng Z, Lugtenberg M, Franse C, Fang X, Hu S, et al. Risk factors and protective factors associated with incident or increase of frailty among community-dwelling older adults: a systematic review of longitudinal studies. PLoS One. 2017;12(6):e0178383.

13. Dohrn IM, Kwak L, Oja P, Sjostrom M, Hagstromer M. Replacing sedentary time with physical activity: a 15-year follow-up of mortality in a national cohort. Clin Epidemiol. 2018;10:179-86.

14. De la Camara MA, Sadarangani KP, Higueras-Fresnillo S, MartinezGomez D, Sanchez-Sanchez JL, et al. Sedentary behaviour, physical activity, and sarcopenia among older adults in the TSHA: isotemporal substitution model. International Journal of Obesity (2005). 2019; 10(1):188-98.

15. Kim M-J. Isotemporal Substitution Analysis of Accelerometer-derived Sedentary Behavior, Physical Activity Time, and Physical Function in Older Women: A Preliminary Study. Exerc Sci. 2015;24(4):373-81.

16. Fried LP. Interventions for human frailty: physical activity as a model. Cold Spring Harbor Perspectives in Medicine. 2016;6(6):1-14.

17. Kehler DS, Hay JL, Stammers AN, Hamm NC, Kimber DE, et al. A systematic review of the association between sedentary behaviors with frailty. Exp Gerontol. 2018;114:1-12.

18. Kehler DS, Theou O. The impact of physical activity and sedentary behaviors on frailty levels. Mech Ageing Dev. 2019;180:29-41.

19. Kowalski K, Rhodes R, Naylor PJ, Tuokko H, MacDonald S. Direct and indirect measurement of physical activity in older adults: a systematic review of the literature. Int J Behav Nutr Phys Act. 2012;9:148.

20. Blodgett J, Theou O, Kirkland S, Andreou P, Rockwood K. The association between sedentary behaviour, moderate-vigorous physical activi- ty and frailty in NHANES cohorts. Maturitas. 2015;80(2):187-91.

21. Del Pozo-Cruz B, Manas A, Martin-Garcia M, Marin-Puyalto J, Garcia-Garcia FJ, et al. Frailty is associated with objectively assessed sedentary behaviour patterns in older adults: Evidence from the Toledo Study for Healthy Aging (TSHA). PloS One. 2017;12(9):e0183911.

22. Kehler DS, Clara I, Hiebert B, Stammers AN, Hay JL, et al. The association between bouts of moderate to vigorous physical activity and patterns of sedentary behavior with frailty. Exp Gerontol. 2018;104:28-34.

23. Kehler DS, Clara I, Hiebert B, Stammers AN, Hay JL, et al. Sex-differences in relation to the association between patterns of physical activity and sedentary behavior with frailty. Arch Gerontol Geriatr. 2019;87: 103972.

24. Manas A, Del Pozo-Cruz B, Guadalupe-Grau A, Marin-Puyalto J, Alfaro-Acha A, et al. Reallocating accelerometer-assessed sedentary time to light or moderate-to-vigorous-intensity physical activity reduces frailty levels in older adults: an isotemporal substitution approach in the TSHA Study. J Am Med Dir Assoc. 2018;19(2):185 e1-e6.

25. Manas A, Pozo-Cruz BD, Rodriguez-Gomez I, Losa-Reyna J, Rodriguez-Manas L, et al. Can physical activity offset the detrimental consequences of sedentary time on frailty? A moderation analysis in 749 older adults measured with accelerometers. J Am Med Dir Assoc. 2019;20(5):634-8.e1

26. Nagai K, Tamaki K, Kusunoki H, Wada Y, Tsuji S, et al. Isotemporal substitution of sedentary time with physical activity and its associations with frailty status. Clin Interv Aging. 2018;13:1831-6.

27. Yuki A, Otsuka R, Tange C, Nishita Y, Tomida M, et al. Daily physical activity predicts frailty development among community-dwelling older Japanese adults. J Am Med Dir Assoc. 2019;1:1-5. 